\section{IDDF2021-ABS-0062 A NOVEL DECISION AID IMPROVES KNOWLEDGE AND QUALITY OF PREGNANCY-RELATED DECISION-MAKING IN IBD}

${ }^{1}$ Joseph Louis Pipicella*, ${ }^{1}$ Neda Karimi, ${ }^{2}$ Grace Wang, ${ }^{3}$ Laura Willmann, ${ }^{1}$ Joseph Descallar, ${ }^{4}$ Katie O'Connor, ${ }^{3}$ Susan Connor, ${ }^{5}$ Yvette Leung, ${ }^{2}$ Vivian Huang, ${ }^{3}$ Astrid-Jane Williams. ${ }^{1}$ The Ingham Institute for Applied Medical Research, Sydney, NSW, Australia; ${ }^{2}$ Department of Medicine, University of Toronto, Toronto, ON, Canada; ${ }^{3}$ Department of Gastroenterology and Hepatology, Liverpool Hospital, Sydney, NSW, Australia; ${ }^{4}$ Division of Gastroenterology, Mount Sinai Hospital, Toronto, ON, Canada; ${ }^{5}$ University of British Columbia, Vancouver, British Columbia, Canada

10.1136/gutjpl-2021-IDDF.126

Background Women with inflammatory bowel disease (IBD) with poor IBD-specific reproductive knowledge experience more voluntary childlessness. This is associated with medication fear, which must be addressed given active IBD during preconception correlates with worse intrapartum disease and poor fetal outcomes. The Pregnancy IBD Decision Aid (PIDA) is an online tool offering personalised decision support on fertility, pregnancy, and medications in IBD (IDDF2021-ABS0062 Figure 1. Screenshot of the Pregnancy IBD Decision Aid tool ). This study aimed to assess PIDA's impact on knowledge and quality of decision-making among preconception and pregnant IBD patients, and to evaluate its feasibility.

Methods Preconception and pregnant patients (18-45yrs) from Canada and Australia completed questionnaires before and after viewing PIDA. Quality of decision-making and IBD-specific pregnancy knowledge were assessed using:

1 Decisional Conflict Scale (DCS)

1 Self-Efficacy Scale (SES)

1 Crohn's and Colitis Pregnancy Knowledge Score (CCPKnow).

Patients and clinicians completed feasibility surveys following PIDA review. Paired t-test assessed PIDA's limited effectiveness.

Results DCS and SES were completed by 42 Crohn's disease and 32 ulcerative colitis patients (preconception: $n=41$; pregnant: $n=33)$. DCS improved for preconception and pregnant patients post-PIDA (effect size $0.44, \mathrm{p}<0.0001$ ). SES improved for preconception patients (effect size 0.32, $\mathrm{p}=0.0001$ ), and in both cohorts CCPKnow also improved $(\mathrm{n}=76$, effect size $0.66, \mathrm{p}<0.0001)$.

Seventy-three patients assessed PIDA's feasibility. PIDA's length $(\mathrm{m}=3.05 \pm 0.44)$, readability $(\mathrm{m}=3.09 \pm 0.5)$ and content

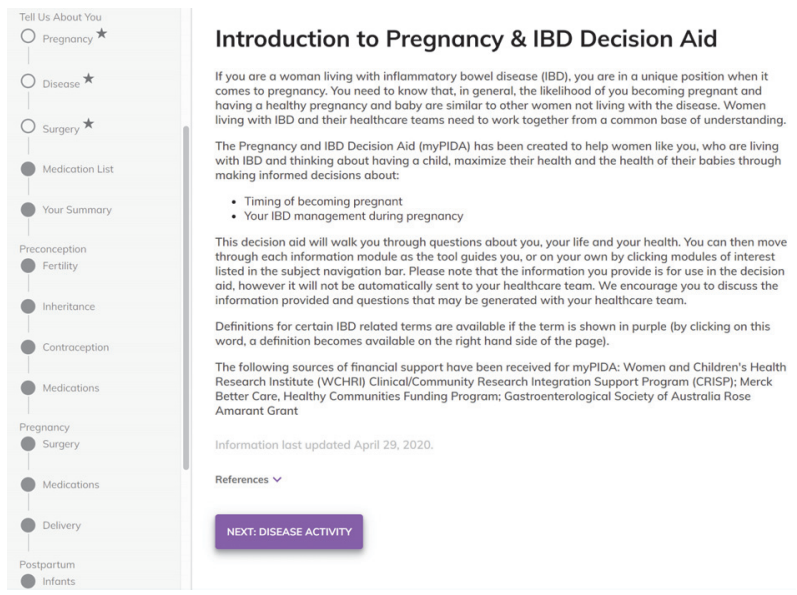

Abstract IDDF2021-ABS-0062 Figure 1 amount $(\mathrm{m}=2.91 \pm 0.81)$ were perceived as appropriate ( $1=$ limited, $5=$ excessive). Perceived usefulness was high among patients $(\mathrm{m}=4.09 \pm 0.93 ; 1=$ least useful, $5=$ most useful). Clinicians $(n=14)$ believed PIDA had appropriate length $(m=3.3$ $\pm 0.6)$, readability $(\mathrm{m}=3.3 \pm 0.8)$, and content amount $(\mathrm{m}=3.4$ $\pm 0.8)$, and deemed PIDA useful for patients $(\mathrm{m}=4.6 \pm 0.8)$ and themselves $(\mathrm{m}=4.8 \pm 0.8)$.

Conclusions PIDA improved patient knowledge and quality of decision-making. Patients developed a strengthened belief in their ability to make informed decisions, and patients/clinicians found PIDA feasible. Therefore, PIDA may reduce voluntary childlessness.

\section{IDDF2021-ABS-0063 USING A CONSENSUS-BASED METHOD TO DEVELOP A NOVEL PAEDIATRIC-SPECIFIC INFLAMMATORY BOWEL DISEASE EHEALTH CLINICAL MANAGEMENT SYSTEM}

${ }^{1}$ Joseph Louis Pipicella*, ${ }^{2}$ Angharad Vernon-Roberts, ${ }^{1}$ Alissa Walsh, ${ }^{1}$ Andre Wierzbicki, ${ }^{2}$ Andrew Day, ${ }^{3}$ David William Carter, ${ }^{4}$ Edward Giles, ${ }^{2}$ Richard Gearry, ${ }^{5}$ Susan Jane Connor, ${ }^{6}$ Jane Mary Andrews, ${ }^{7}$ Shoma Dutt. ${ }^{1}$ Crohn's Colitis Cure, Sydney, NSW, Australia; ${ }^{2}$ Department of Paediatrics, University of Otago Christchurch, Christchurch, New Zealand; ${ }^{3}$ Stratos Technology Partners, Christchurch, New Zealand; ${ }^{4}$ Department of Paediatrics, Monash University, Clayton, VIC, Australia; ${ }^{5}$ Department of Gastroenterology and Hepatology, Liverpool Hospital, Sydney, NSW, Australia; ${ }^{6}$ Central Adelaide Local Health Network, Adelaide, SA, Australia; 'The Children's Hospital at Westmead Clinical School, University of Sydney, Sydney, Australia

\subsection{6/gutjil-2021-IDDF.127}

Background 'CCCare' is an inflammatory bowel disease (IBD)specific electronic medical record (eMR) developed to support care for adults with IBD. 'CCCare-P' is the paediatric 'version' of CCCare. CCCare-P may provide coordination of care between paediatric and adult centres during transition. A consensus method, described here, was developed prior to CCCare-P build to ensure necessary inclusions were incorporated. We aim to examine this method's effectiveness.

Methods A consensus group (CG) comprising adult and paediatric health care professionals was formed. The CG created a list of potential functionalities (e.g. ability to score disease activity) and supporting resources (e.g. Pediatric Ulcerative Colitis Activity Index [PUCAI]). The CG met and reviewed this list, identifying common themes. The CG was divided into three working groups (WG) based on identified themes (WG1=Diagnosis and management; $\underline{W G 2}=$ Paediatric chronic disease; $\underline{\mathrm{WG} 3}=$ 'Non-clinical' items). Two voting rounds (VR1 \& VR2) used the same Likert scale $(0=$ Omit, $4=$ Essential) to determine opinions on proposed functionalities and resources. VR1 occurred after an initial item review meeting. WGs then independently reviewed functionalities and supporting resources, and later presented their findings to the CG in an online, two-day meeting. After circulating meeting minutes, attendees completed VR2. The content validity index method (CVI) was used to judge which functionalities and resources reached consensus (CVI $0.78)$.

Results All five WG1 functionalities reached consensus in both voting rounds. The consensus process led to additional resources being included in VR2, with the number deemed necessary for inclusion in VR2 being 38 (68\% consensus) compared to 23 in VR1 (65\% consensus). All five WG2 functionalities and 11/15 resources reached consensus in VR2. For WG3, 4/8 functionalities and 3/28 resources reached consensus in VR1; 